\title{
3D Myocardial Contraction Imaging Based on Dynamic Grid Interpolation: Theory and Simulation Analysis
}

\author{
Shuhui Bu Non-member (bushuhui@mibel.cs.tsukuba.ac.jp) \\ Tsuyoshi Shiina Member (shiina@cs.tsukuba.ac.jp) \\ Makoto Yamakawa Member (yamakawa@mibel.cs.tsukuba.ac.jp) \\ Hotaka Takizawa Member (takizawa@cs.tsukuba.ac.jp)
}

Keywords : left ventricle, myocardial contraction function, deformable model, combined autocorrelation, weighted phase gradient

Accurate assessment of local myocardial contraction is important for diagnosis of ischemic heart disease because decrases of myocardial motion often appears in the early stage of the disease.

However, conventional approaches such as tissue Doppler imaging and strain-rate imaging techniques cannot provide us enough quantitative information about local myocardial contraction. In order to resolve the problem, a myocardial strain imaging system, which enables us to precisely evaluate the myocardial contraction, has been proposed previously.

In this research, we improve the quality of myocardial strain images by two improvements: 1) improve the phase-shift accuracy by incorporating extended combined autocorrelation method (ECAM) into weighted phase gradient method (WPGM); 2) improve the strain image's accuracy and contrast by using 2-D dynamic grid interpolation (DGI). From the simulation results, we can conclude that the strain image's accuracy and contrast can be improved.

In this 3-D myocardial strain imaging system, a 2-D transducer is used for acquiring echo data from myocardium by volumetric sector scan. Radio frequency (RF) signals for each scan line are received at all elements of the transducer. The phase-shifts at every measuring point between two consecutive frames are calculated by the ECAM. The displacement vectors are calculated by the WPGM from obtained phase-shifts. At the same time, the endocardium and epicardium boundaries are segmented from the B-mode image, and the myocardial meshes are generated from the myocardial boundaries. Next, the un-smoothed displacement vectors and myocardial meshes are input to displacement re-sampling module. The displacement vectors in all mesh's nodes are calculated by bilinear interpolation, and their coordinates are also translated from Cartesian coordinates to cylinder coordinates. In this research, the 2-D DGI method is used for smoothing the displacement vectors. By this method, the displacement vectors are restricted by virtual springs. If adjacent two nodes' displacements are larger than other nodes' displacements, the adjusted new displacement will be decreased. Therefore, the overall error can be reduced. Finally, the strain tensors are calculated from the smoothed displacement vectors.

The performances of the 3-D myocardial strain imaging are evaluated by numerically simulating the short axis imaging of a 3-D myocardial model. By this simulation model, both radial and twisting motions can be simulated. Two group simulations are performed. In each group, at the post-processing two displacement smoothing filters are applied: normal smoothing filters (median and averaging filters) and 2-D DGI. Radial strain in normal wall is set to $2.0 \%$, and $0.6 \%$ in ill contraction region. The RF signals have a signal-to-noise ratio (SNR) of $20 \mathrm{~dB}$.

The resulting images of simulation model II are shown in Figure 1. From the resulting images and profiles, we can see that the accuracy and contrast of resulting image $1(\mathrm{~F})$ which is generated by the 2-D DGI and ECAM are best. Especially at the endocardium or epicardium boundaries, the strain values which are generated by the 2-D DGI and ECAM are more accurate compared with those generated by other methods. Therefore, the abnormal myocardial contraction region can be clearly identified.

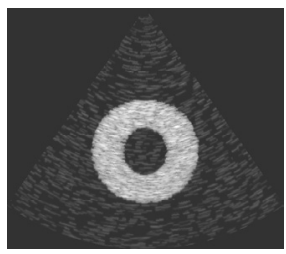

(A) B-Mode image

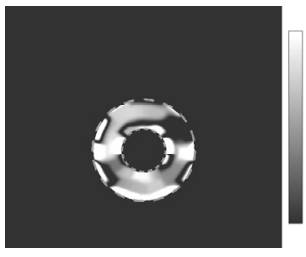

(C) NS, CAM (case 1)

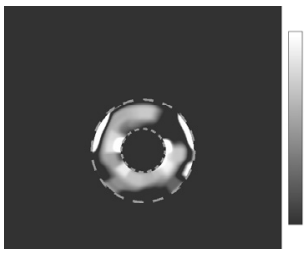

(E) NS, ECAM (case 3)

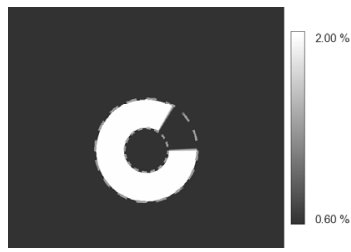

(B) Ideal radial strain

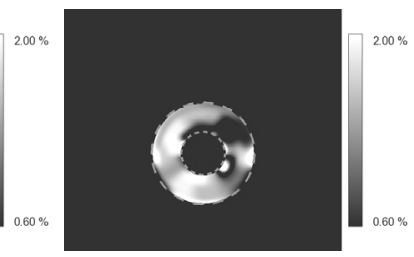

(D) 2D DGI, CAM (case 2)

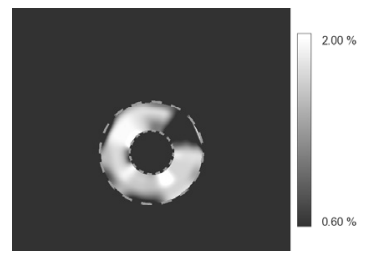

(F) 2D DGI, ECAM (case 4)

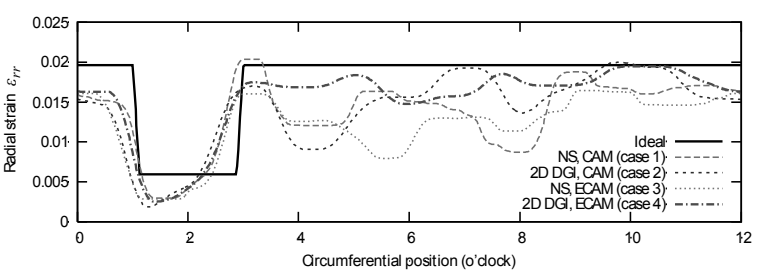

(G) Profiles

Fig. 1. Resulting images and profiles of simulation model II 


\title{
3D Myocardial Contraction Imaging Based on Dynamic Grid Interpolation: Theory and Simulation Analysis
}

\author{
Shuhui Bu* \\ Tsuyoshi Shiina* Member \\ Makoto Yamakawa* Member \\ Hotaka Takizawa* Member
}

\begin{abstract}
Accurate assessment of local myocardial contraction is important for diagnosis of ischemic heart disease, because decreases of myocardial motion often appear in the early stages of the disease. Three-dimensional (3-D) assessment of the stiffness distribution is required for accurate diagnosis of ischemic heart disease. Since myocardium motion occurs radially within the left ventricle wall and the ultrasound beam propagates axially, conventional approaches, such as tissue Doppler imaging and strain-rate imaging techniques, cannot provide us with enough quantitative information about local myocardial contraction. In order to resolve this problem, we propose a novel myocardial contraction imaging system which utilizes the weighted phase gradient method, the extended combined autocorrelation method, and the dynamic grid interpolation (DGI) method. From the simulation results, we conclude that the strain image's accuracy and contrast have been improved by the proposed method.
\end{abstract}

Keywords: left ventricle, myocardial contraction function, deformable model, combined autocorrelation, weighted phase gradient

\section{Introduction}

Heart disease is increasing in the developed countries, and it is almost the main leading cause of death. The primary goal of cardiac imaging and image analysis is to assess the regional functions of the left ventricle (LV) of heart. From these regional functions, the quantitative estimates of the location and extent of ischemic myocardial injury can be obtained. Regional left ventricular deformation can be determined using the principal imaging modalities, including echocardiography, radionuclide imaging, computed tomography $(\mathrm{CT})$, and magnetic resonance (MR) imaging.

The widely used echocardiography methods are BMode imaging, M-Mode imaging, tissue Doppler imaging $(\mathrm{TDI})^{(1)-(4)}$, and strain-rate imaging $(\mathrm{SRI})^{(5)-(9)}$. The shape of ventricles can be visualized by B-mode imaging, but quantitative diagnosis information cannot be obtained. Through M-mode imaging, a specified scan line's myocardial motion can be visualized within a period of time. But only the beam direction's motion information can be obtained. The last two methods also provide motion information only in the beam direction ${ }^{(10)(11)}$. Furthermore, if the motion is larger than one quarter of a wave-length, aliasing may occur and accurate myocardial strain cannot be acquired. There are some other methods, which have been put on much research effort. For example, Pa-

\footnotetext{
* University of Tsukuba, Graduate School of System and Information Engineering,

1-1-1 Tennoudai, Tsukuba, Ibaraki 305-8573
}

pademetris et al. proposed a method ${ }^{(12)}$ by which myocardial boundaries are extracted from B-mode images. Then the myocardial strain tensors are calculated by applying the finite element method (FEM). But this method suffers from a critical drawback. It requires accurate segmentation results, so that the image segmentation must be processed by inspectors. Hence, it is not convenient and the processing speed is not fast. In order to solve the above problems, a myocardial strain imaging system based on the combined autocorrelation method $(\mathrm{CAM})^{(13)}$ and the $3-\mathrm{D}$ weighted phase gradient method (WPGM) ${ }^{(14)(15)}$, which can detect all directions' true displacement vectors, has been proposed previously $^{(16)-(18)}$.

In this research, in order to obtain a more accurate phase-shift, a new method which incorporates an extended combined autocorrelation method (ECAM) (19) (20) into the WPGM is proposed. The ECAM was originally proposed for detecting phase-shift and lateral motion simultaneously in tissue elasticity measuring. In real heart motion, myocardium has complex and relatively large deformations, especially the twisting deformation during contraction. Generally, the CAM only detects the beam direction's motion, but under large or complex deformations, large errors will occur. More accurate results have been obtained from the simulations which use ECAM as the phase-shift detector. Therefore, we can conclude that ECAM is more suitable in myocardial strain imaging.

Calculating spatial derivation of displacement is a necessary step in strain calculation, but the numerical cal- 
culation is fairly sensitive to noise. To overcome this problem, smoothing filters must be applied before the strain calculation. In this research, a two-dimensional (2-D) dynamic grid interpolation (DGI) method, which is based on local myocardial motion tracking method ${ }^{(21)}$ developed by Ohyama et al., is proposed. By applying this displacement smoothing filter, we can see that the smoothing effects are better than those obtained utilizing normal smoothing (NS) filters (median and averaging filters). The accuracy and contrast of resulting images have been greatly improved. Therefore, the ill contraction region can be clearly identified.

This paper is organized as follows. We present the principle of the proposed 3-D myocardial strain imaging firstly in section 1 . In section 2, the deformable model of LV is provided. The simulations which we performed will be elaborated in Section 4. Then we discuss our overall findings in Section 5. Finally, the conclusions of our work are given.

\section{3-D Myocardial Strain Imaging}

The 3-D myocardial strain imaging system consists of the following seven parts: transducer, phase-shift detector, weighted phase gradient method based displacement calculating, image segmentation and myocardium meshing, displacement re-sampling, displacement smoothing, and strain calculation. The measurement coordinate system and processing flow of this system are illustrated in Fig. 1.

In this system, a 2-D transducer is used for acquiring echo data from the myocardium by volumetric sector scanning. Radio frequency (RF) signals for each scan line are received at all elements in the probe. The phase-shifts at every measuring point between two consecutive frames are calculated by the $\mathrm{CAM}^{(13)}$ or the ECAM $^{(19)(20)}$, and then the displacement vectors are calculated by WPGM ${ }^{(14)(15)}$ from obtained phase-shifts. At the same time, the B-mode image, which is generated by the RF signals obtained from the aperture in the center transducer, is acquired. From the B-mode image, the myocardial endocardium boundaries are segmented ${ }^{(22)}$, while the epicardium boundary is calculated from the endocardium boundary by adding a fixed radius. Next, the myocardium meshes are generated from the myocardial boundaries. In the following step, the un-smoothed displacement vectors and myocardial meshes are input into a displacement re-sampling module. The displacement vectors in each mesh's node are calculated by bilinear interpolation ${ }^{(23)}$, and their coordinates are also translated from Cartesian coordinates to cylinder coordinates. After this, the DGI method is used for smoothing the displacement vectors. Finally, the strain tensors are calculated from the smoothed displacement vectors.

2.1 Weighted Phase Gradient Method The weighted phase gradient method ${ }^{(14)(15)}$ is one type of spatial compounding methods. The displacement vectors are compounded by phase-shifts which are obtained from a 2-D array transducer. The measurement coordinate system is illustrated in Fig. 1. During the myocardial contracting period, ultrasound beams are radiated from the origin point $O$ on the 2-D array aperture to scatterers around the measuring point $P$, and the scattering waves are received at all elements on the 2-D array aperture before beam forming. The scatterers at the measuring point $P$ have the displacement vectors $\left(u_{x}, u_{y}, u_{z}\right)$. The phase-shift $\phi(x, 0, z, t)$ between successive scattering waves received by the aperture $Q(x, 0, z)$, which is induced by each deformation, is represented by the following equation ${ }^{(14)}$ :

$$
\begin{aligned}
\frac{\lambda}{2 \pi} \phi(x, 0, z, t)= & \frac{\lambda}{4 \pi}\left(1+\frac{L}{R}\right) \phi(0,0,0, t) \\
& -\frac{u_{x} \cdot x+u_{z} \cdot z}{R}, \cdots . .
\end{aligned}
$$

where $\lambda$ is the wavelength, time $t$ corresponds to $2 L / c$, $c$ is the speed of sound in tissues, $L$ is the distance from the origin $O$ to measuring point $P$, and $R$ is the distance from $P$ to each receiving element $Q$. The phase-shift $\phi(0,0,0, t)$ and $\phi(x, 0, z, t)$ can be detected by autocorrelation techniques at points $O$ and $Q(x, 0, z)$, respectively. To obtain more accurate phase-shifts, CAM or ECAM methods need to be incorporated.

The displacement components $u_{x}$ and $u_{z}$ can be calculated from equation (1) by employing the least-squares method as follows:

$$
\begin{aligned}
& u_{x}=\frac{\sum_{k=1}^{N} x_{k} \mu\left(x_{k}, 0, z_{k}, t\right)}{\sum_{k=1}^{N} x_{k}^{2}}, \\
& u_{z}=\frac{\sum_{k=1}^{N} z_{k} \mu\left(x_{k}, 0, z_{k}, t\right)}{\sum_{k=1}^{N} z_{k}^{2}}, \cdots
\end{aligned}
$$

where

$$
\begin{aligned}
\mu(x, 0, z, t)= & \frac{\lambda}{4 \pi}(L+R) \phi(0,0,0, t) \\
& -\frac{\lambda}{2 \pi} R \phi(x, 0, z, t), \cdots \ldots \ldots \ldots .
\end{aligned}
$$

and $N$ is the number of all receiving apertures.

After $u_{x}$ and $u_{z}$ are obtained, $u_{y}$ can be calculated by ${ }^{(14)}$ :

$$
\begin{aligned}
u_{y}= & -u_{x} \tan \alpha \cos \beta-u_{z} \tan \alpha \sin \beta \\
& +\frac{\lambda}{4 \pi} \frac{\phi(0,0,0, t)}{\cos \alpha}, \ldots \ldots \ldots \ldots \ldots
\end{aligned}
$$

where $\alpha$ is the angle between the radiated ultrasound beam and $y$ axis, and $\beta$ is the angle between the plane which is created by the beam and $y$ axis and the $x-y$ plane.

2.2 Extended Combined Autocorrelation Method Under large deformation, the phase-shift $\phi$ at $Q(x, 0, z)$ or $O$ may be affected by aliasing. Therefore, an accurate phase-shift cannot be obtained through a conventional autocorrelation method. To address this problem, we incorporate $\mathrm{CAM}^{(13)}$ or $\mathrm{ECAM}^{(19)(20)}$ into the WPGM as a phase-shift detector. Compared with the CAM, ECAM can detect beam direction's motion and lateral motion simultaneously. Because the search range of ECAM has been enlarged, the detected phaseshifts are more stable compared with those detected by CAM. In our research, because only axial phase-shifts 




Fig. 1. Measurement coordinate system of the 2-D array aperture in the WPGM, and the data processing flow.

are used in WPGM, the detected lateral motions will be neglected.

$s_{1}(x, 0, z, t, 0)$ and $s_{2}(x, 0, z, t, l)$ are regarded as analytic signals after the quadrature demodulation of $\mathrm{RF}$ signals acquired under pre- and post-deformation at receiving aperture $Q$, respectively. Firstly, the crosscorrelation function $R_{s}(x, 0, z, t ; n, l)$ between $s_{1}(x, 0, z$, $t, 0)$ and $s_{2}(x, 0, z, t+n T / 2, l)$ which delays the postdeformation signal $s_{2}$ by $n$ times half a period $T$ of the reference ultrasound wave, is calculated as follows ${ }^{(19)}$ :

$$
\begin{aligned}
& R_{s}(x, 0, z, t ; n, l) \\
& \quad=R_{u}(x, 0, z, t ; n, l) e^{-j \Phi(x, 0, z, t ; n, l)},
\end{aligned}
$$

where $R_{u}$ indicates the correlation function of the envelopes of pre- and post-deformation signals, and $l$ is lateral shift. Next, the argument $\Phi(x, 0, z, t ; n, l)$ of $R_{s}$, which corresponds to the phase-shift at each $n$ step and $l$ lateral shift, is obtained as follows ${ }^{(19)}$ :

$$
\begin{aligned}
\Phi(x, 0, z, t ; n, l) & =-\arg \left\{R_{s}(x, 0, z, t ; n, l)\right\} \\
& =\phi(x, 0, z, t)-n \pi, \cdots \cdots \cdots
\end{aligned}
$$

where $n$ is a integer, and $n=-M, \ldots,-1,0,1, \ldots, N$. $l . l$ is also a integer, and $l=-E, \ldots,-1,0,1, \ldots, F$.

The true phase-shift $\phi(x, 0, z, t)$ can be obtained by selecting a correct $n$ and $l$. The correlation coefficient $C(x, 0, z, t ; n, l)$ between $s_{1}(x, 0, z, t, 0)$ and $s_{2}(x, 0, z, t+$ $n T / 2, l)$ is calculated simultaneously as:

$$
\begin{aligned}
C & (x, 0, z, t ; n, l) \\
& =\frac{\left|R_{u}(x, 0, z, t ; n, l)\right|}{\sqrt{\left|R_{s}(x, 0, z, t ; 0,0)\right|\left|R_{s}(x, 0, z, t ; n, l)\right|}} .
\end{aligned}
$$$$
\ldots \ldots \ldots \ldots \ldots \ldots+(8)
$$

The above processing indicates that $(M+N+1) \times$
$(E+F+1)$ pairs of $\{\Phi(x, 0, z, t ; n, l), C(x, 0, z, t ; n, l)\}$ are derived at a time $t$. By determining the correct $n=k$ and $l=p$ which maximize $C(x, 0, z, t ; n, l)$, the true phase-shift $\phi(x, 0, z, t)$ is obtained by the following equation:

$$
\phi(x, 0, z, t)=\Phi(x, 0, z, t ; k, p)+k \pi . \cdots \cdots \cdots
$$

2.3 Dynamic Grid Interpolation Numerical calculation of the displacement gradient is very sensitive to noise. Therefore, smoothing filters must be applied before calculating the displacement gradient. In previous researches ${ }^{(16)-(18)}$, median and averaging smoothing filters have been applied. These two filters have the serious drawback that the strain value's dynamic range may be decreased, which means the strain image's contrast may also be decreased. Because the smoothing effects in all positions are the same, detailed information may be lost. Therefore, the boundaries are not clear. To improve the contrast of strain images and resolution, a dynamic grid interpolating method, which is based on the local myocardial motion tracking method ${ }^{(21)}$, is proposed for displacement smoothing. By comparing the results, we can see that the strain images' quality have been greatly improved by using our method.

The detail procedure can be described as follows. The first step is creating a mesh which represents the the region of interest (ROI). In our research, the myocardium is segmented, and its endocardium boundaries are also segmented ${ }^{(22)}$. The epicardium boundary is calculated from endocardium boundary by adding a fixed radius. Then the myocardium are divided into quad elements along the radial and circumference directions equally.

In the second step, the displacement vectors are resampled to generate mesh nodes by using the bilinear 
interpolation method. The displacement vectors array $\vec{u}=\left(u_{r}, u_{t}\right)$ in all sampling nodes is defined by the following formula:

$$
\begin{gathered}
\overrightarrow{\mathbf{u}}=\left[\vec{u}^{1,1}, \vec{u}^{1,2}, \cdots, \vec{u}^{1, m}\right. \\
\vec{u}^{2,1}, \vec{u}^{2,2}, \cdots, \vec{u}^{2, m} \\
\cdots \cdots \cdots \\
\left.\vec{u}^{n, 1}, \vec{u}^{n, 2}, \cdots, \vec{u}^{n, m}\right]^{T}
\end{gathered}
$$

where $m$ represents the radial direction's node number, and $n$ represents the circumference direction's node number. The revised displacement vectors array is defined as the same form of $\overrightarrow{\mathbf{u}}$, and its definition is:

$$
\overrightarrow{\mathbf{u}}^{\prime}=\overrightarrow{\mathbf{u}}+\vec{\epsilon}, \ldots \ldots \ldots \ldots \ldots \ldots \ldots \ldots \ldots \ldots \ldots \ldots \ldots \ldots \ldots
$$

where $\vec{\epsilon}=\left(\epsilon_{\mathbf{r}}, \epsilon_{\mathbf{t}}\right)$ is the revising displacement vectors array for all nodes. The definition of displacement revising vectors array $\vec{\epsilon}$ have the same form as (10).

Here, we define an error function which represents the overall difference between original and revised displacement. By minimizing the error function, the revised displacement vectors can be calculated as follows:

$$
\begin{aligned}
e\left(\epsilon_{\mathbf{r}}, \epsilon_{\mathbf{t}}\right)= & \sum_{i=1}^{n} \sum_{j=1}^{m}\left[\left(\epsilon_{r}^{i, j}\right)^{2}+\left(\epsilon_{t}^{i, j}\right)^{2}\right] \\
& +\frac{E_{r r}}{2} \sum_{i=1}^{n} \sum_{j=1}^{m-1}\left(u_{r}^{\prime i, j+1}-u_{r}^{\prime i, j}\right)^{2} \\
& +\frac{G_{r t}}{2} \sum_{i=1}^{n-1} \sum_{j=1}^{m-1}\left[\left(u_{r}{ }^{i+1, j+1}-u_{r}{ }^{i+1, j}\right)\right. \\
& \left.-\left(u_{r}^{\prime}{ }^{i, j+1}-u_{r}{ }^{i, j}\right)\right]^{2} \\
& +\frac{E_{t t}}{2} \sum_{i=1}^{n-1} \sum_{j=1}^{m}\left(u_{t}^{\prime}{ }^{i+1, j}-u_{t}{ }^{i, j}\right)^{2} \\
& +\frac{G_{t r}}{2} \sum_{i=1}^{n-1} \sum_{j=1}^{m-1}\left[\left(u_{t}^{i+1, j+1}-u_{t}{ }^{i, j+1}\right)\right. \\
& \left.-\left(u_{t}^{\prime}{ }^{i+1, j}-u_{t}^{i, j}\right)\right]^{2} \ldots \ldots \ldots
\end{aligned}
$$

$E_{r r}$ and $E_{t t}$ are the pseudo-elasticity parameters which control axial displacement revision. If adjacent two nodes' displacements are larger than other nodes, the adjusted new displacements will be decreased. The great elasticity parameter $E$ makes the node's extra large movement difficult, which means the error movement can be restrained, while $G_{r t}$ and $G_{t r}$ are pseudo shear elasticity parameters. They control the shear direction's revising effect.

When the error function is minimized, the following equations can be derived:

$$
\begin{aligned}
& \frac{\partial e\left(\epsilon_{\mathbf{r}}, \epsilon_{\mathbf{t}}\right)}{\partial \epsilon_{\mathbf{r}}}=\mathbf{A}_{\mathbf{r}} \epsilon_{\mathbf{r}}-\mathbf{b}_{\mathbf{r}}=0 \\
& \frac{\partial e\left(\epsilon_{\mathbf{r}}, \epsilon_{\mathbf{t}}\right)}{\partial \epsilon_{\mathbf{t}}}=\mathbf{A}_{\mathbf{t}} \epsilon_{\mathbf{t}}-\mathbf{b}_{\mathbf{t}}=0
\end{aligned}
$$

The matrix $\mathbf{A}_{\mathbf{r}}$ and vectors array $\mathbf{b}_{\mathbf{r}}$ in the radial direction can be obtained by deriving equation (12) at each



Fig. 2. Illustration of mesh definition in dynamic grid interpolation method and displacement vectors.

node in the mesh, and the matrix $\mathbf{A}_{\mathbf{t}}$ and vectors array $\mathbf{b}_{\mathbf{t}}$ can also be obtained by the same way. To obtain the displacement revising vectors array $\epsilon_{\mathbf{r}}$ and $\epsilon_{\mathbf{t}}$, the best optimized elastic parameters $E_{r r}, G_{r t}, E_{t t}$, and $G_{t r}$ are manually selected from preliminary experiments. Because the linear equations are very large, LAPACK ${ }^{(24)}$ is used for solving them.

In the current research stage, only the radial strain $\varepsilon_{r r}$ is assessed. Therefore, only the radial displacement $u_{r}$ is smoothed. To obtain revised radial displacement $u_{r}$, only equation (13) is solved. Because $\partial e\left(\epsilon_{\mathbf{r}}, \epsilon_{\mathbf{t}}\right) / \partial \epsilon_{\mathbf{r}}$ is only correlated to parameters $E_{r r}$ and $G_{r t}, E_{t t}$ and $G_{t r}$ are neglected in this research.

2.4 Computation of Strain Since most myocardial wall motion is radially deformed on the short axis view of the left ventricle, radial strain can be used to assess the wall stiffness. In this research, cylindrical coordinates are used in calculating strain tensor. The radial strain tensor $\varepsilon_{r r}$ is defined as ${ }^{(25)}$ :

$$
\varepsilon_{r r}=\frac{\partial u_{r}}{\partial r}
$$

In the previous formulation (15), the radial displacement $u_{r}$ is calculated by:

$$
u_{r}=u_{x} \cos \theta+u_{y} \sin \theta
$$

where $u_{x}$ and $u_{y}$ are the displacements calculated from the WPGM, and $\theta$ is the measured point's angle as illustrated in Fig. 3.

\section{Deformable Model of LV}

To evaluate our myocardial strain imaging, the LV deformable model ${ }^{(26)(27)}$ is used for generating the myocardial shape model. The volumetric deformable LV models and parameter functions are represented by meshes at specified time phases of the cardiac cycle. The LV 
model's coordinates are defined as $\xi=(p, r, w)$, where $p$ spans from the apex to the base of the LV, $r$ goes out from the endocardial surface to epicardial surface, and $w$ covers from septum, anterior, lateral, and posterior of the LV. The defined coordinates are shown in Fig. 4. The Cartesian coordinates $\mathbf{x}(\xi, t)=(x(\xi, t), y(\xi, t), z(\xi, t))^{T}$ are used for representing a time-varying model.

At time step $t$, the model can be expressed by Cartesian coordinates as ${ }^{(26)}$ :

$$
\mathbf{x}_{t}=\mathbf{x}_{0}+\mathbf{s}+\mathbf{T r}
$$

where $\mathbf{x}_{t}$ is the model at time $t, \mathbf{x}_{0}$ is the original model before deformation, and $\mathbf{s}$ is the displacement vector at time step $t$. $\operatorname{Tr}$ is the model's overall translation vector.

3.1 Model Parameters The deformable model has four parameters, as listed in Table 1.

The parameters $\alpha_{1}$ and $\alpha_{2}$ are the aspect ratios along the $x$ - and $y$-axes of the LV model, respectively. In order to simplify the computation, the parameters $\alpha_{1}$ and $\alpha_{2}$ can be combined to a single parameter, $\alpha$, which models the radial contraction of the LV.

By using the model parameters, the displacement vector $\mathbf{s}$ in equation (17) can be expressed as ${ }^{(26)}$ :

$$
\mathbf{s}=\left(\begin{array}{c}
e_{1} \cos (\tau(\xi))-e_{2} \sin (\tau(\xi)) \\
e_{1} \sin (\tau(\xi))+e_{2} \cos (\tau(\xi)) \\
e_{3}
\end{array}\right), \ldots \ldots
$$

where

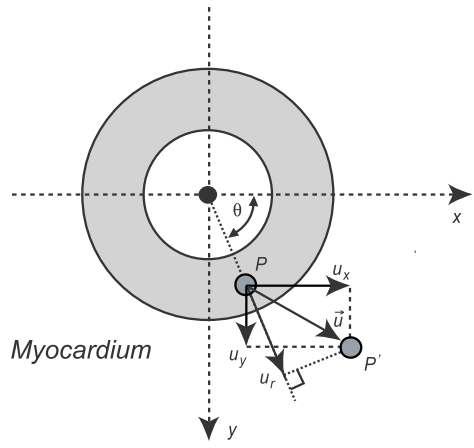

Fig. 3. Coordinates definition for radial displacement calculation.
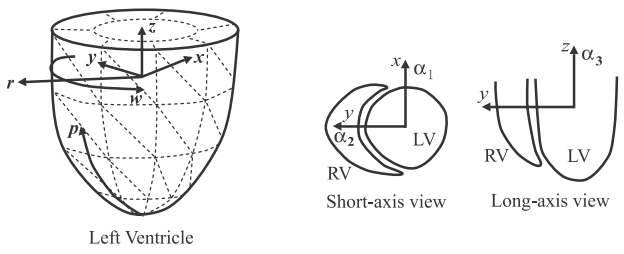

Fig. 4. Definition of an LV deformable model's coordinates system $^{(26)}$.

Table 1. Model parameters of the LV deformable model.

\begin{tabular}{ll}
\hline \hline Parameters & Representation \\
\hline$\alpha_{1}, \alpha_{2}(\alpha)$ & Radial contractions \\
$\alpha_{3}$ & Longitudinal contraction \\
$\tau$ & Twisting about the long axis \\
\hline \hline
\end{tabular}

$$
\begin{aligned}
& e_{1}=a_{0} r^{\prime} \alpha(\xi) \cos p \cos w \\
& e_{2}=a_{0} r^{\prime} \alpha(\xi) \cos p \sin w \\
& e_{3}=a_{0} r^{\prime} \alpha_{3}(\xi) \sin p .
\end{aligned}
$$

$r^{\prime}$ is the distance between the origin point (apex) in the LV coordinates system and the point of $\xi . a_{0}$ is an extra scale which is applied to $r^{\prime}$. In this research, $a_{0}$ is set to 1.

\section{Simulation Analysis}

The performances of the 3 -D myocardial strain imaging are evaluated by numerically simulating the shortaxis imaging of a 3-D LV model, as illustrated in Fig. $5(\mathrm{C})$. In this research, three group comparison simulations are performed. In each group, both CAM and ECAM phase-shift detectors are used, and two displacement smoothing filters are applied in the postprocessing: normal smoothing filters (median \& averaging filters) and 2-D DGI.

\subsection{Simulation Conditions The LV deforma-} tion model is used for generating the LV shapes before and after deformation. The model's pre- and post- deformation are illustrated in Figs. 5(A), (B). The myo-



(A)

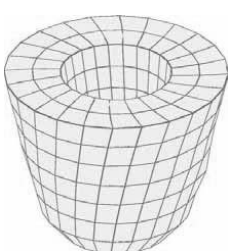

(B)

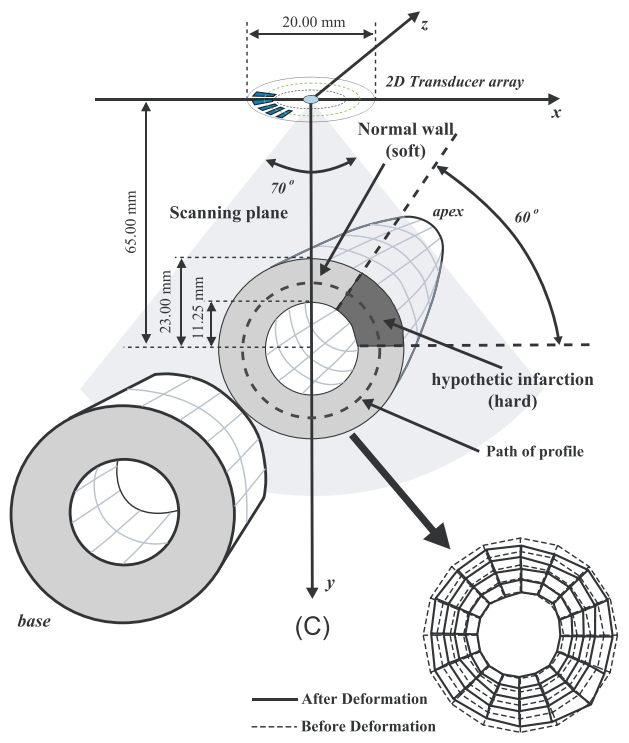

(D) Meshes in 2D DGI

Fig. 5. Meshes of simulation model and measuring condition (simulation case II). (A) Mesh of original LV model. (B) Mesh of deformed LV (deformations have been magnified). (C) Illustration of measuring condition (simulation model II), scanning plane, and the path of profile. (D) Illustration of meshes in 2-D DGI (simulation model II). The solid line indicates the post-deformation (radial motions have been magnified), while the dash line indicates the pre-deformation. 
cardium inner radius is $11.25 \mathrm{~mm}$, and the outer radius is $23 \mathrm{~mm}$. From Kerckhoffs et al.'s research ${ }^{(28)}$, we find that during the systole period, the total radial strain is about $20 \%$, and the twisting angle is about 10 degrees. Normally, the systole period's time is about $1 / 3$ of a cardiac cycle. Therefore, the average twisting between two adjacent frames is about 1 degree $(0.0174 \mathrm{rad})$ when the frame rate is 30 frame-per-seconds (FPS), and the radial strain in normal wall is about $2 \%$. The stiffness of the infarction is set to be about two times greater than that of the surrounding normal wall, because this stiffness ratio is generally referred to as the typical stiffness ratio of the infarction to normal wall ${ }^{(9)}$. To realize the stiffness in the infarcted region is three times that in the normal region, the radial strain $\varepsilon_{r r}$ in the infarcted region is set to be one third of that in the normal region. Therefore, the ill contraction region's radial strain is set to $0.6 \%$. The overall translation vector $\mathbf{T r}$ in equation (17) is set to $(0.5,0.5,0.0)^{T} \mathrm{~mm}$.

By applying the above simulation model's parameters to each mesh node in the deformable LV model, a postdeformation model can be obtained. The myocardium is frequently considered as an incompressible body and the myocardial motion consists of the displacements due to deformation and translation ${ }^{(9)}$. Therefore, the scatterers' positions are relocated according to the postdeformation results. Next, the simulating RF signals are generated from the scatterer field.

The 2-D transducer's out diameter is $20 \mathrm{~mm}$. The ultrasonic pulse has a center frequency of $3.75 \mathrm{MHz}$, and the fractional bandwidth is $40 \%$. The scanning plane is located between apex and base under short-axis view. The scan plane and hypothetic infarction are illustrated in Fig. 5(C). The scanning frame rate is $30 \mathrm{FPS}$. In these simulations, the RF signals with a signal-to-noise ratio (SNR) of $20 \mathrm{~dB}$ are digitized at a rate of $20 \mathrm{MHz}$. The median filter's window size and averaging filter's window size are the same as our pervious researches ${ }^{(16)-(18)}$, and their values are $W_{m}=3.5 \mathrm{~mm}$ and $W_{a}=3.5 \mathrm{~mm}$, respectively. The element numbers in the radial direction are 80, and 256 in the circumferential direction.

4.2 Evaluation Parameters For quantitative analysis of the result images' quality, we define several evaluation parameters: standard deviation $\sigma$, standard deviation $\sigma_{I}$ of distribution between ideal value and measured, and contrast $C$, as follows:

$$
\begin{aligned}
\sigma & =\frac{\sqrt{\frac{\sum_{k}\left(x_{k}-\bar{x}\right)^{2}}{N-1}}}{I_{\max }-I_{\min }} \times 100 \%, \\
\sigma_{I} & =\frac{\sqrt{\frac{\sum_{k}\left(x_{k}-x_{I}\right)^{2}}{N-1}}}{I_{\max }-I_{\min }} \times 100 \%, \\
C & =\frac{\bar{V}_{\max }-\bar{V}_{\min }}{I_{\max }-I_{\min }} \times 100 \%,
\end{aligned}
$$

where $x_{k}$ is the measured value, $\bar{x}$ is the mean value of measured, $x_{I}$ is the ideal value, $\bar{V}_{\text {max }}$ and $\bar{V}_{\text {min }}$ are the maximum and minimum mean measured values, respectively, $I_{\max }$ and $I_{\min }$ are maximum and minimum ideal values, and $N$ is the data number.

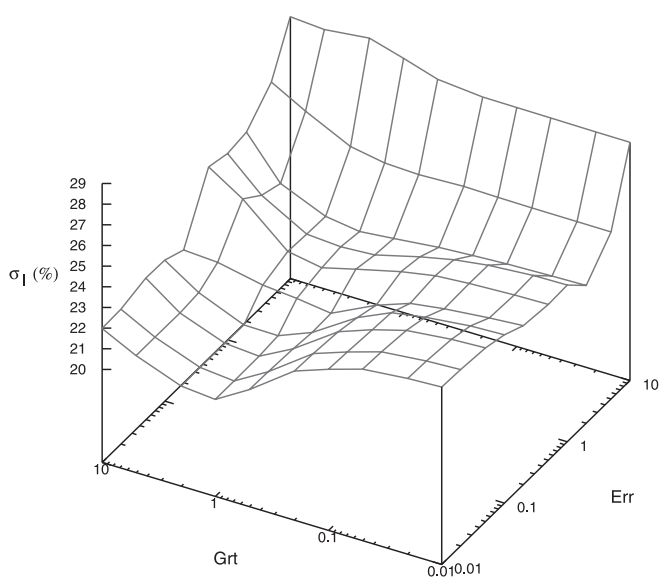

(A)

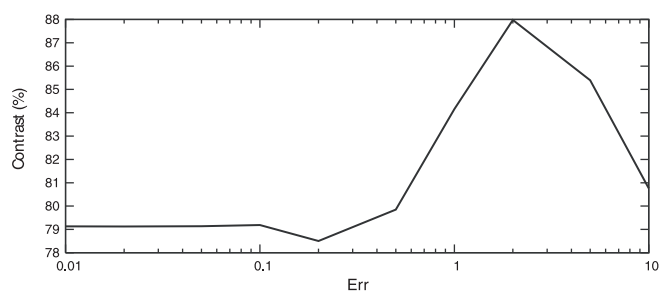

(B)

Fig. 6. (A) The accuracy $\sigma_{I}$ when different 2-D DGI parameters are used. (B) The contrast $\mathrm{C}$ by using different $E_{r r}$ when $G_{r t}=1.0$.

4.3 Parameter Selection The 2-D DGI's parameters are selected from experiments. In the preliminary experiments, the parameters $E_{r r}$ and $G_{r t}$ are both selected from the following values: $[0.01,0.02,0.05,0.1$, $0.2,0.5,1.0,2.0,5.0,10.0]$. The distributions of accuracy $\sigma_{I}$, which is defined in formula $(21)$, are plotted in Fig. 6(A) by using different values for $E_{r r}$ and $G_{r t}$. From this figure, we can see that the accuracy is best when $G_{r t}=1.0$. In this research, not only the accuracy is considered for selecting the parameters, the contrast is also an important evaluation parameter. Therefore, in order to obtain the performance of contrast when different parameters are used, the figure for the contrast $C$, which is defined in formula (22), is plotted in Fig. 6(B) by using different $E_{r r}$ when $G_{r t}=1.0$. From this figure, we can see that the contrast is best when $E_{r r}$ is around 1.0 to 5.0. But the accuracy is not good when $E_{r r}$ is bigger than 1.0. As a consequence, for obtaining a good balance between accuracy and contrast, $E_{r r}=1.0$ and $G_{r t}=1.0$ are selected as the optimal parameters for the following simulation analyses.

4.4 Evaluating Angle Dependency In order to evaluate the proposed method's angle dependency, two models which have different infraction positions are simulated. In model I, the infarcted wall is located around 5 to 7 o'clock, where its radial motion and ultrasound beam are same. Under this condition, the abnormal wall's motion can be detected by both TDI and SRI. In model II, the infarcted wall is located around 1 to 3 o'clock. It is well known that both TDI and SRI cannot detect correct wall motion in this region ${ }^{(1)-(4)}$. 


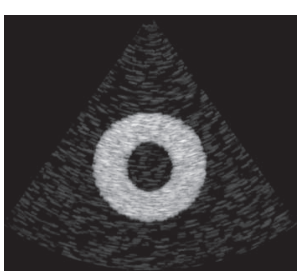

(A) B-Mode image

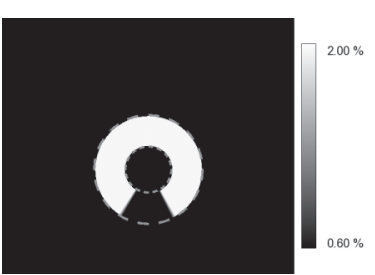

(B) Ideal result

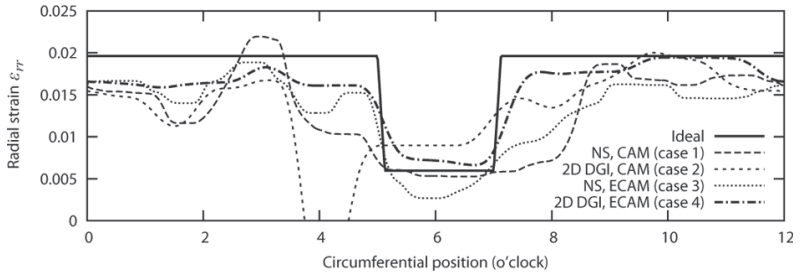

(C) Profiles

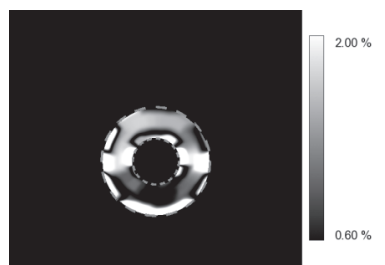

(D) NS, CAM (case 1)

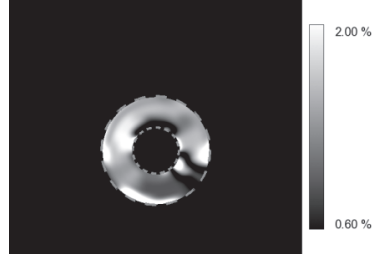

(E) 2-D DGI, CAM (case 2)

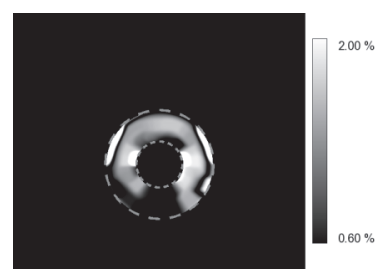

(F) NS, ECAM (case 3)

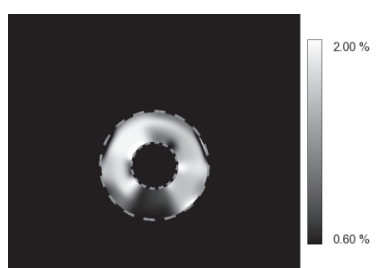

(G) 2-D DGI, ECAM (case 4)

Fig. 7. Resulting images and profiles of simulation model I.

Table 2. Performance comparison of model I and II.

\begin{tabular}{ccccccc}
\hline \hline Simulation Model & Case & Phase-shift Detector & Smoothing Filter & $\sigma$ & $\sigma_{I}$ & Contrast $(C)$ \\
\hline I & 1 & CAM & Median and averaging & $27.75 \%$ & $44.79 \%$ & $62.27 \%$ \\
I & 2 & CAM & 2-D DGI & $40.41 \%$ & $62.80 \%$ & $53.10 \%$ \\
I & 3 & ECAM & Median and averaging & $14.92 \%$ & $36.69 \%$ & $71.54 \%$ \\
I & 4 & ECAM & 2-D DGI & $8.99 \%$ & $19.90 \%$ & $77.05 \%$ \\
\hline II & 1 & CAM & Median and averaging & $22.35 \%$ & $39.04 \%$ & $62.79 \%$ \\
II & 2 & CAM & 2-D DGI & $23.98 \%$ & $37.32 \%$ & $69.10 \%$ \\
II & 3 & ECAM & Median and averaging & $19.48 \%$ & $47.09 \%$ & $54.04 \%$ \\
II & 4 & ECAM & 2-D DGI & $14.14 \%$ & $23.19 \%$ & $83.08 \%$ \\
\hline \hline
\end{tabular}

The resulting images from simulation model I are shown in Fig. 7. Fig. 7(A) shows the B-mode image. Fig. $7(\mathrm{~B})$ shows the ideal radial strain $\varepsilon_{r r}$. Fig. 7 (D) shows the radial strain generated by NS and CAM, (E) shows the radial strain generated by 2-D DGI and CAM, (F) shows the radial strain generated by NS and ECAM, and $(\mathrm{G})$ shows the radial strain generated by 2D DGI and ECAM. The profiles of comparison between the 4 cases is shown in Fig. $7(\mathrm{C})$, and the profiles' path is shown in Fig. $5(\mathrm{C})$. The same applied to model II, the resulting images for this simulation are show in Fig. 8 , respectively. The quantitative results for these two models are summarized in Table 2 .

From the resulting images, we can see that our proposed method does not have the problem of angle dependency. There are no distinct accuracy decreases in model II compared with model I.

\subsection{Evaluating Infarction Size's Influence}

The performances of our method for the models where the infarctions have different sizes are also simulated. In model III, the location of the infarcted wall is around 5.30 to 6.30 o'clock (30 degrees). In model IV, the infarcted wall is located around 1.30 to 2.30 o'clock (30 degrees). In model $\mathrm{V}$, the infarcted wall is located around 4 to 8 o'clock (120 degrees). And in model VI, the infarcted wall is located around 0 to 4 o'clock (120 degrees).

The resulting images for each model are shown in Figs. 9-12, respectively, and the quantitative results for these four models are summarized in Table 3.

In simulation models III and IV, although the in- farcted wall's size is small, by our proposed method, the infarcted wall can also be correctly detected. The number of false low contraction regions has been reduced. In simulation models $\mathrm{V}$ and VI, because the size of the infarcted wall is large, large areas of low contraction can be clearly identified. With ECAM and 2-D DGI, the accuracy is at its best. In addition, the number of false low contraction regions is also reduced.

4.6 Evaluating the Radial Direction's Strain Distribution Usually, ischemic heart diseases first occur in the endocardium. The infarction is gradually spreaded from endocardium to epicardium. In order to response this status, in the following two models, the radial strain is gradually increased with the radius increasing. The radial strain's distribution in the radial direction is linear. The radial strain in the endocardium is $0.6 \%$, and $2.0 \%$ in the epicardium. In model VII, the infarcted wall is located around 5 to 7 o'clock. In model VIII, the infarcted wall is located around 1 to 3 o'clock.

The resulting images for each model are shown in Figs. 13 and 14, respectively, and the quantitative results for these four models are summarized in Table 3.

In the last two simulation models VII and VIII, the radial strain's value range is low. Therefore, it is difficult to detect the abnormal wall. The ill contraction regions are hard to be recognized by old methods, but the strain patterns, which are generated by ECAM and 2-D DGI, are closest to ideal patterns.

\section{Discussion}

In our research, we have made two main improve- 
Table 3. Performance comparison of model III, IV, V, VI, VII, and VIII.

\begin{tabular}{|c|c|c|c|c|c|c|}
\hline$\overline{\text { Simulation Model }}$ & Case & Phase-shift Detector & Smoothing Filter & $\overline{\sigma \sigma}$ & $\overline{\sigma_{I}}$ & Contrast $(C)$ \\
\hline III & 1 & CAM & Median and averaging & $22.03 \%$ & $39.50 \%$ & $43.52 \%$ \\
\hline III & 2 & CAM & 2-D DGI & $16.28 \%$ & $27.37 \%$ & $52.84 \%$ \\
\hline III & 3 & ECAM & Median and averaging & $17.26 \%$ & $40.08 \%$ & $65.36 \%$ \\
\hline III & 4 & ECAM & 2-D DGI & $13.84 \%$ & $24.15 \%$ & $77.01 \%$ \\
\hline IV & 1 & CAM & Median and averaging & $21.04 \%$ & $37.91 \%$ & $52.83 \%$ \\
\hline IV & 2 & CAM & 2-D DGI & $9.18 \%$ & $25.36 \%$ & $52.77 \%$ \\
\hline IV & 3 & ECAM & Median and averaging & $17.27 \%$ & $43.67 \%$ & $62.40 \%$ \\
\hline IV & 4 & ECAM & 2-D DGI & $14.66 \%$ & $24.72 \%$ & $56.18 \%$ \\
\hline $\mathrm{V}$ & 1 & CAM & Median and averaging & $22.18 \%$ & $34.45 \%$ & $77.90 \%$ \\
\hline V & 2 & CAM & 2-D DGI & $15.84 \%$ & $34.19 \%$ & $70.66 \%$ \\
\hline $\mathrm{V}$ & 3 & ECAM & Median and averaging & $17.06 \%$ & $35.56 \%$ & $67.94 \%$ \\
\hline V & 4 & ECAM & 2-D DGI & $12.95 \%$ & $21.63 \%$ & $77.70 \%$ \\
\hline VI & 1 & CAM & Median and averaging & $26.12 \%$ & $45.74 \%$ & $53.81 \%$ \\
\hline VI & 2 & CAM & 2-D DGI & $20.23 \%$ & $25.47 \%$ & $68.30 \%$ \\
\hline VI & 3 & ECAM & Median and averaging & $25.39 \%$ & $40.76 \%$ & $65.13 \%$ \\
\hline VI & 4 & ECAM & 2-D DGI & $20.80 \%$ & $22.51 \%$ & $100.00 \%$ \\
\hline VII & 1 & CAM & Median and averaging & $62.39 \%$ & $105.12 \%$ & $12.94 \%$ \\
\hline VII & 2 & CAM & 2-D DGI & $38.38 \%$ & $67.31 \%$ & $50.02 \%$ \\
\hline VII & 3 & ECAM & Median and averaging & $37.56 \%$ & $100.54 \%$ & $21.60 \%$ \\
\hline VII & 4 & ECAM & 2-D DGI & $18.03 \%$ & $42.61 \%$ & $80.12 \%$ \\
\hline VIII & 1 & CAM & Median and averaging & $52.46 \%$ & $93.65 \%$ & $18.98 \%$ \\
\hline VIII & 2 & CAM & 2-D DGI & $20.66 \%$ & $61.18 \%$ & $55.92 \%$ \\
\hline VIII & 3 & ECAM & Median and averaging & $40.93 \%$ & $108.28 \%$ & $39.00 \%$ \\
\hline VIII & 4 & ECAM & 2-D DGI & $27.00 \%$ & $56.70 \%$ & $59.79 \%$ \\
\hline
\end{tabular}

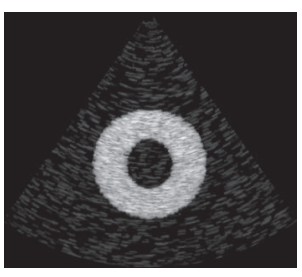

(A) B-Mode image

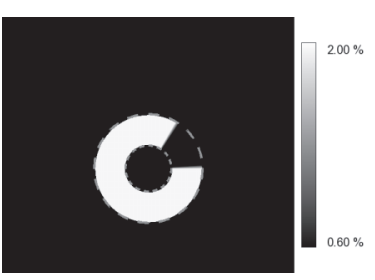

(B) Ideal result

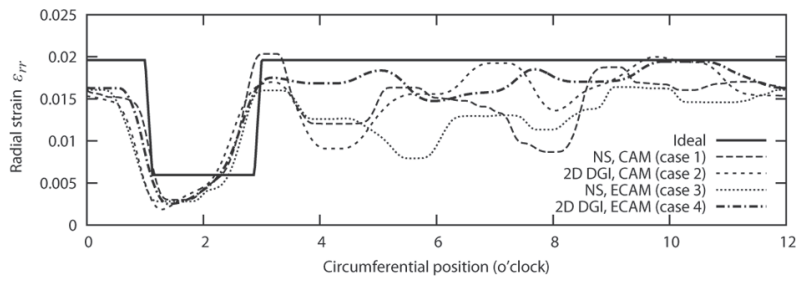

(C) Profiles

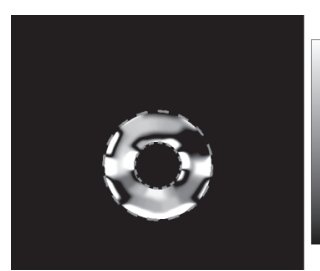

(D) NS, CAM (case 1)

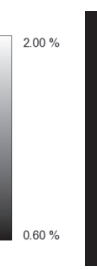

(E) 2-D DGI, CAM (case 2)

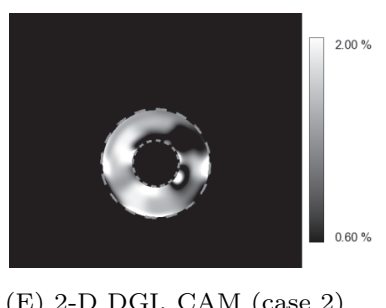

Fig. 8. Resulting images and profiles of simulation model II.

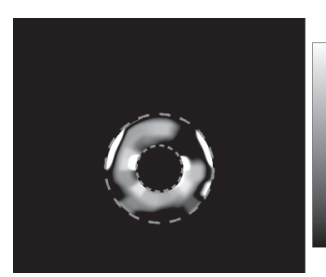

(F) NS, ECAM (case 3)

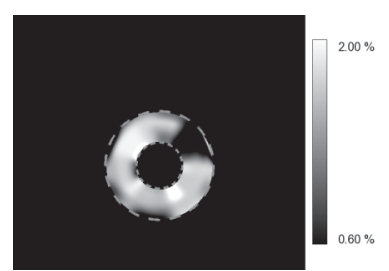

(G) 2-D DGI, ECAM (case 4) ments in myocardial contraction imaging: 1) improve the phase-shift accuracy by incorporating ECAM into WPGM; 2) improve the strain image's accuracy and contrast by using 2-D DGI.

The main advantage of ECAM compared with CAM is the expansion of the search range from 1-D to 2-D. As a result, large and complex motion can be detected. Because the myocardium has complex and large deformations, the ECAM is more suitable for phase-shift detecting than the CAM. In addition, more accurate and stable results can be achieved. While large twisting is applied to the LV model and noise is applied to RF signals, the accuracy and contrast of the resulting images which are generated by the CAM are not good. However, an ECAM-based method can output more accurate results so that the infarcted region can be clearly identified. For example, in model II, there is a region around 4 o'clock for endocardium where its strain values have big errors, as shown in Figs. 8(D) and (E). However, the strain values' accuracy in this region has been improved by using the ECAM, as shown in Figs. 8(F) and $(\mathrm{G})$.

The second improvement in our myocardial contraction imaging is applying 2-D DGI for smoothing displacement vectors. The strain calculation is fairly sensitive to noise. A small fluctuation in displacement can bring a bigger fluctuation in the strain result when the spatial resolution is high. Therefore, the errors are magnified. Of course, this problem can be roughly resolved by increasing the step size for the displacement difference, but it causes low spatial resolution in strain results.

In traditional approaches, the median and averaging filters are cooperated for removing noise and smoothing. The median filter is used for reducing speckle noise without blurring the strains. But each of these two filters has a drawback. The window size is usually set to the same value for every point. If the window size is 


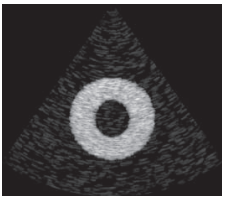

(A) B-Mode image

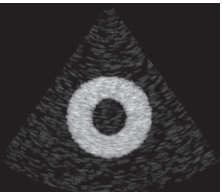

(A) B-Mode image

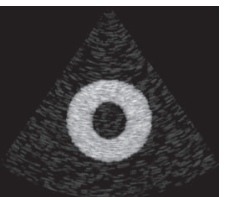

(A) B-Mode image

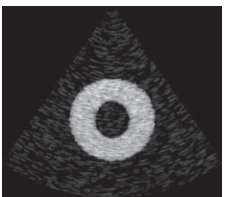

(A) B-Mode image

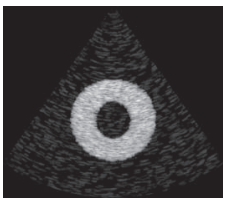

(A) B-Mode image

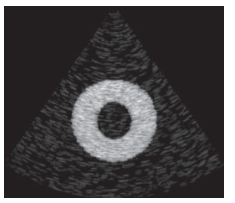

(A) B-Mode image

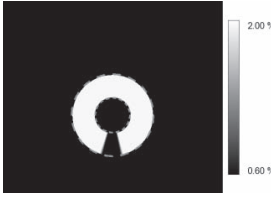

(B) Ideal result

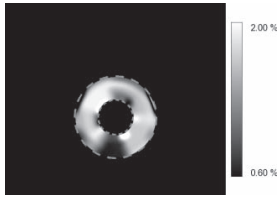

(C) 2-D DGI, ECAM

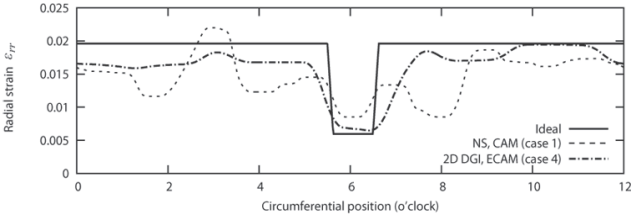

(D) Profiles

Fig. 9. Resulting images and profiles of simulation model III.

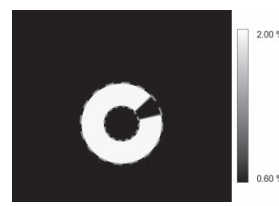

(B) Ideal result

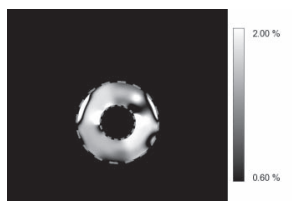

(C) 2-D DGI, ECAM

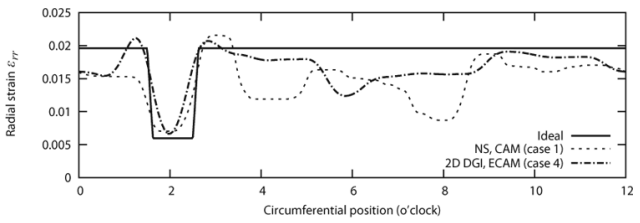

(D) Profiles

Fig. 10. Resulting images and profiles of simulation model IV.

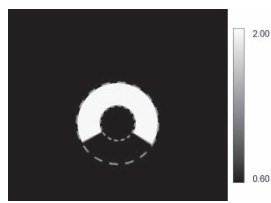

(B) Ideal result

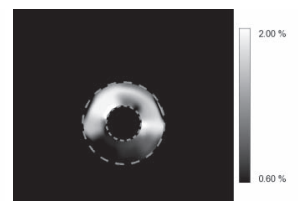

(C) 2-D DGI, ECAM

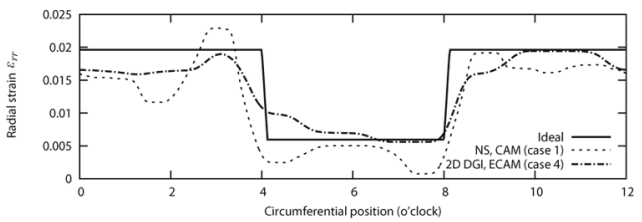

(D) Profiles

Fig. 11. Resulting images and profiles of simulation model V.

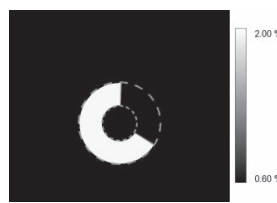

(B) Ideal result



(C) 2-D DGI, ECAM



(D) Profiles

Fig. 12. Resulting images and profiles of simulation model VI.

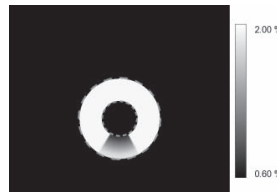

B) Ideal result



(C) 2-D DGI, ECAM

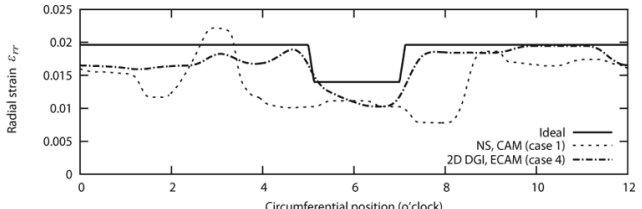

(D) Profiles

Fig. 13. Resulting images and profiles of simulation model VII.

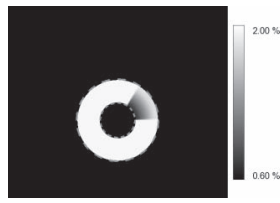

(B) Ideal result

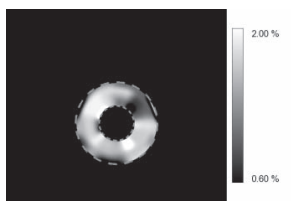

(C) 2-D DGI, ECAM

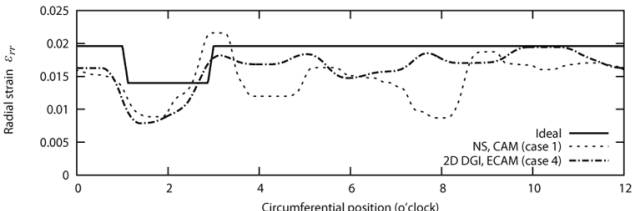

(D) Profiles

Fig. 14. Resulting images and profiles of simulation model VIII.

set to be a large value, the contrast will decrease, and spatial resolution will decrease. On the contrary, if the window size is set be a small value, the filters cannot handle large area's speckle noise, and the fluctuation in strain images will be great.

The DGI method can overcome the above drawback. Therefore, a excellent balance between spatial resolution and accuracy can be achieved. In this method, an overall error function is used for calculating the revis- ing displacements. If some areas' errors are large, the revising effect will be great. On the contrary, if some areas' errors are small, the revising effect will likewise be small. Compared with median and averaging filter, there are some considerations of the mechanics of relationship in this method.

From the resulting images and profiles, we can see that it provides better resolution and accuracy compared with those generated by normal smoothing filters. 
The profiles show that the errors of the 2-D DGI and the ECAM have been greatly decreased compared with those generated by normal smoothing filters. In all simulation models, the boundaries between normal walls and abnormal walls, which are generated by the 2-D DGI and the ECAM, are clearer than those generated by other methods. Since, the strain images' patterns which are generated by the 2-D DGI and the ECAM are closer to ideal patterns, and the images' contrasts are also improved compared with other methods, our proposed method is expected to provide more accurate diagnosis information.

\section{Conclusion}

The simulation experiments which utilize the ECAM as WPGM's phase-shift detector have been performed, and better results are obtained. The median and averaging smoothing filters are replaced by a dynamic grid interpolation filter. We also applied the deformable model to a 3-D LV wall motion simulation. By this simulation model, we provided three group comparisons. From the comparison results, we can conclude that the accuracy and contrast of resulting images have been improved by using ECAM and 2-D DGI method. Therefore, the ill myocardial contraction region can be clearly identified.

Although better results are obtained, there are still some works that need to be done in the future. Firstly, 2D DGI has been implemented, but we consider whether further improvements can be achieved. Currently, the 2D DGI's parameters $E_{r r}$ and $G_{r t}$ are manually selected. In the next researches, an automatic parameter selecting algorithm will be performed, and more accurate results are expected. Secondly, in this research, the displacement smoothing is just processed between 2 adjacent frames. In the following researches, we consider the situation that the 2-D DGI will be extended so that more than 2 frames' data can be processed. More stable strain results are expected as a result.

(Manuscript received Feb. 1, 2007,

revised June 12, 2007)

\section{References}

(1) W.M. McDicken, G.R. Sutherland, C.M. Moran, and L.N. Gordon: "Colour Dppler velocity imaging of the myocardium", Ultrasound Med. Biol., Vol.18, No.61, pp.651-654 (1992)

(2) N. Yamazaki, Y. Mine, A. Sano, M. Hirama, K. Miyatake, M. Yamagishi, and N. Tanaka: "Analysis of ventricular wall motion using color-corded tissue Doppler imaging system", Jpan. J. Appl. Phys., Vol.33, No.5, pp.3141-3146 (1994)

( 3 ) G.R. Sutherland, M.J. Stewart, and K.W.E. Groundstroem: "Color Doppler myocardial imaging: A new technique for assessment of myocardial function", J. Amer. Soc. Echocardiogr., Vol.7, pp.441-458, Sep.-Oct. (1994)

(4) T. Edvardsen, S. Urheim, H. Skulstad, K. Steine, H. Ihlen, and O.A. Smiseth: "Quantification of left ventricular systolic function by tissue doppler echocardiography", Circulation, Vol.105 (17), pp.2071-2077 (2002)

(5) A. Heimdal, A. Stoyle, H. Torp, and T. Skjaerpe: "Real-time strain rate imaging of the left ventricle by ultrasound", $J$. Amer. Soc. Echocardiogr., Vol.11, pp.1013-1019, Nov. (1998)

(6) J. D'hooge, E. Konofagou, F. Jamal, A. Heimdal, L. Barrios,
B. Bijnens, J. Thoen, F. Van de Werf, G. Sutherland, and P. Suetens: "Two-dimensional ultrasonic strain rate measurement of the humanheart in vivo", IEEE Trans. Ultrason. Ferroelectr. \& Freq. Control, Vol.49(2), pp.281-286 (2002)

( 7 ) J. D'hooge, A. Heimdal, F. Jamal, T. Kukulski, B. Bijnens, F. Rademakers, L. Hatle, P. Suetens, and G. R. Sutherland: "Regional strain and strain rate measurements by cardiac ultrasound: principles, implementation and limitations", Eur. J. Echocardiography, Vol.1, pp.154-170 (2000)

(8) T.P. Abraham and R.A. Nishimura: "Strain rate imaging for assessment of regional myocardial function", American Heart Association, pp.1403-1406 (2002)

(9) K. Kaluzynski, X. Chen, S.Y. Emelianov, A.R. Skovoroda, and M. O'Donnell: "Strain rate imaging using twodimensional speckle tracking," IEEE Trans. Ultrason. Ferroelectr. E Freq. Control, Vol.48(4), pp.1111-1123 (2001)

(10) A. Heimdal, T. Abraham, C. Pislaru, and M. Belohlavek: "Angle dependency of strain rate imaging in an animal model", J. Amer. Soc. Echocardiogr., Vol.13, p.484 (2000)

(11) P.L. Castro, N.L. Greenberg, J. Drinko, M.J. Garcia, and J.D. Thomas: "Potential pitfalls of strain rate imaging: Angle dependency", Biomed. Sci. Instrum., Vol.36, pp.197-202 (2000)

(12) X. Papademetris, A.J. Sinusas, D.P. Dione, and J.S. Duncan: "Estimation of 3D left ventricular deformation from echocardiography", Medical Image Analysis, No.5, pp.17-28, 2001.

(13) T. Shiina, M.M. Doyel, and J.C. Bamber: "Strain imaging using combined RF and envelop autocorrelation processing", IEEE Ultrasonic symposium, pp.1331-1336 (1996)

(14) N. Nitta and T. Shiina: "Real-time three-dimensional velocity vector measurement using the weighted phase gradient method", Jpn. J. Appl. Phys., Vol.37, part 1, No.5B, pp.30583063, May (1998)

(15) N. Nitta and T. Shiina: "A method of tissue elasticity estimation based on three-dimensional displacement vector", Jpn. J. Appl. Phys., Vol.39, part 1, No.5B, pp.3225-3229, May (2000)

(16) N. Nitta, M. Yamakawa, and T. Shiina: "Discussion of 2D strain imaging method", The 24th Symposium on Ultrasonic Electronics, P3-J-57, pp.337-338 (2003)

(17) N. Nitta and T. Shiina: "Myocardial strain imaging based on three-dimensional motion tracking", IEEE Ultrasonic symposium, pp.1258-1261 (2003)

(18) N. Nitta and T. Shiina: "Myocardial strain imaging based on two-dimensional displacement vector measurement", Jpn. J. Appl. Phys., Vol.43, No.5B, pp.3249-3255 (2004)

(19) M. Yamakawa and T. Shiina: "Strain estimation using the extended combined autocorrelation method", Jpn. J. Appl. Phys., Vol.40, No.5B, pp.3872-3876 (2001)

(20) M. Yamakawa, N. Nitta, T. Shiina, T. Matsumura, S. Tamano, T. Mitake, and E. Ueno: "High-speed freehand tissue elasticity imaging for breast diagnosis", Jpn. J. Appl. Phys., Vol.42, No.5b, pp.3265-3270 (2003)

(21) W. Ohyama, N.B. Ismail, T. Warabrashi, F. Kimura, S. Tsuruoka, and K. Sekioka: "Local myocardial motion tracking based on correlation weighted phase difference method", IEICE (A), Vol.J86-A, No.9, pp.917-928, Sep. (2003) (In Japanese)

(22) C. Corsi, M. Borsari, F. Consegnati, A. Sarti, C. Lamberti, A. Travaglini, T. Shiota, and J.D. Thomas: "Left ventricular endocardial surface detection based on realtime 3D echocardiographic data", European Journal of Ultrasound, Vol.13, pp.41-45 (2001)

(23) W.H. Press, S.A. Teukolsky, W.T. Vetterling, and B.P. Flannery: Numerical Recipes in C, Cambridge university press

(24) E. Anderson, Z. Bai, C. Bischof, S. Blackford, J. Demmel, J. Dongarra, J.D. Croz, A. Greenbaum, S. Hammarling, A. McKenney, and D. Sorensen: LAPACK Users' Guide, Third Edition, Society for Industrial and Applied Mathematics, Philadelphia, PA (1999)

(25) Y.C. Fung: A first course in Continum mechanics, PrenticeHall, Inc., Englewood Cliffs, New Jersey, U.S.A.

(26) J. Park, D. Metaxas, and L. Axel: "Analysis of left ventricular wall motion based on volumetric deformable models and MRI-SPAMM", Medical Image Analysis, Vol.1, No.1, pp.5371 (1996)

(27) J. Park and S. Park: "Strain analysis and visualization: left ventricle of a heart", Computer \&s Graphics, Vol.24, pp.701- 
$714(2000)$

(28) R.C.P. Kerckhoffs, P.H.M. Bovendeerd, J.C.S. Kotte, F.W. Prinzen, K. Smits, and T. Arts: "Homogeneity of cardiac contraction despite physiological asynchrony of depolarization: a model study", Annals of Biomedical Engineering, Vol.31, pp.536-547 (2003).

Shuihui Bu (Non-member) received the B.S. degree in en-

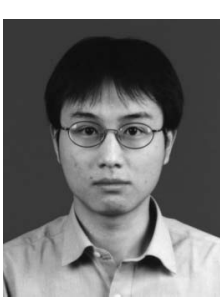
gineering mechanics from Hunan University, Changsha, P.R. China in 2001. He was a graduate student since April 2004 at the Computer Science department, the Graduate School of Systems and Information Engineering, University of Tsukuba, Japan, where he received the M.S. degree in March 2006. He is currently a Ph.D. candidate at the same department. His research interests include the ultrasound imaging, medical imaging, and image processing.

Tsuyoshi Shiina (Member) was graduated from the Elec-


tronic Engineering Department, the University of Tokyo, Tokyo, Japan, in 1982. He received the $\mathrm{MS}$ and $\mathrm{PhD}$ degrees in electronic engineering in 1984 and 1987, respectively, from the University of Tokyo. From 1987 to 1992, he was Lecturer and Associate Professor at the Department of Electronic Engineering, Tokyo University of Agriculture and Technology, Tokyo, Japan. In 1992, he moved to the University of Tsukuba, and he has been a Professor of the University of Tsukuba since 2001. From 1995 to 1996, he was with the institute of Cancer Research and the Royal Marsden NHS Trust in UK as Visiting Professor. His current research interests include visualization technique of structural and functional bio-information, for example, ultrasonic elasticity imaging, 3D blood-flow measurement, and bio-signal processing based on EEG. He was awarded the distinguished paper prize about his research on elasticity imaging by the Japan Society of Ultrasonics in Medicine, 2000. He was also awarded the novel technology prize about Real-Time Tissue Elasticity Imaging System which was produced by cooperation with Hitachi Medical Corporation by Japan Society of Medical Electronics and Biological Engineering, 2005. He is Chair of Medical and Biological Engineering Technical Committees of IEEJ. $\mathrm{He}$ is also Executive Trustee of the Japan Society of Ultrasonics in Medicine.
Makoto Yamakawa (Member) received the M. S. and Ph.

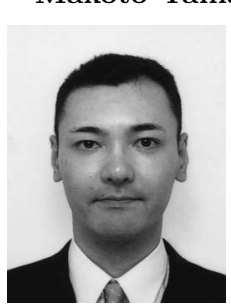
D. degrees from University of Tsukuba, Japan, in 1999 and 2002, respectively. He was a research associate in the Institute of Information Sciences and Electronics, University of Tsukuba from 2002 to 2004, and was promoted to a lecturer in 2005 . He is currently a lecturer in the Graduate School of Systems and Information Engineering, University of Tsukuba. He has been engaged in research on medical engineering, such as visualization of biological signals and medical ultrasonic measurement. He is a member of IEICE, Japan Society of Ultrasonics in Medicine, Japanese Society for Medical and Biological Engineering, and a member of the editorial committee of IEEJ.

Hotaka Takizawa (Member) received his B.Eng., M.Eng.

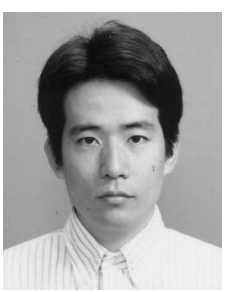
and Ph.D. degrees at the Department of Mechanical Engineering for Computer-Controlled Machinery in 1993, 1995 and 1998, respectively, all from Osaka University, Osaka, Japan. From April 1998 to April 2005, he was a Research Associate at the Department of Knowledge-based Information Engineering, Toyohashi University of Technology, Aichi, Japan. From June 2003 to May 2004, he was a Visiting Research Associate at the University of Chicago, Illinois, USA. Currently, he is a Assistant Professor at the Department of Computer Science, University of Tsukuba, Ibaraki, Japan. He received the Academic Encouragement Award of the Institute of Electronics, Information and Communication Engineers in 2001 and the Best Paper Award of the Japanese Society of Medical Imaging Technology in 2004. His research interests include pattern recognition, computer vision and artificial intelligence. He is a member of the Institute of Electrical and Electronics Engineers, the Institute of Electronics, Information and Communication Engineers, the Information Processing Society of Japan, the Japanese Society for Medical and Biological Engineering. 